\title{
Paul Trap and the Problem of Quantum Stability
}

\author{
L. Chotorlishvili , K. Nickoladze and G. Mchedlishvili \\ Physics Department of the Tbilisi State University, \\ Chavchavadze av.3,0128, Tbilisi,Georgia and \\ Email: lchotor33@yahoo.com
}

(Dated: August 5, 2021)

\begin{abstract}
This work is devoted to the investigation of possibility of controlling of ions motion inside Paul trap. It has been shown that by proper selection of the parameters of controlling electric fields, stable localization of ions inside Paul trap is possible. Quantum consideration of this problem is reduced to the investigation of the Mathieu-Schrodinger equation. It has been shown that quantum consideration is appreciably different from classical one that leads to stronger limitations of the values of the parameters of stable motion. Connection between the problem under study and the possibility of experimental observation of quantum chaos has been shown.

PACS numbers: 73.23.-b,78.67.-n,72.15.Lh,42.65.Re
\end{abstract}




\section{INTRODUCTION}

It is well known that formation of Bose-Einstein condensate is based on application of miniature trap technology [1, 2, 3, 4, 5, 6].

Formation of condensates of alkaline atoms turned out to be possible thanks to unification of new ideas and technologies developed in other areas of physics. First of all magnetic trap must be formed in order to confine the condensate.

In spite of this, the traps formed by application of variable electric fields are the subjects of special interests. The point is that, the confinement of separate ions in trap for a long time gives new possibilities in laser spectroscopy. In addition, single entrapped ion represents itself a unique object to prove the fundamental laws of quantum mechanics [7, 8, 9, 10, 11]. For example, single ion placed inside Paul trap is applied to realize a quantum gate, and a chain consisting of many ions placed in linear trap may be regarded as promising component of future quantum computer [12].

The goal of this work is the study of quantum dynamics based on the application of Paul trap [13, 14, 15, 16].

Not going into details that can be found in [16], we shall describe shortly necessary information about Paul trap needed further.

Paul trap is based exceptionally on the application of sign-variable electric field. Besides, potential formed by electrodes inside trap has the following form [16]:

$$
\phi(r, t)=f(t)\left(x^{2}+y^{2} z^{2}-2 z^{2}\right)
$$

where

$$
f(t)=\frac{U+V \cos \left(\omega_{r f} t\right)}{r_{0}^{2}+2 z_{0}^{2}} .
$$

Here, $U$ and $V$ are the amplitudes of constant and variable fields, $r_{0}$-radius of circular electrode, $2 z_{0}$-distance between covers, and $\omega_{r f}=2 \pi / T$ frequency of variable field.

The ion's classical motion in potential (1) is described by:

$$
M \ddot{\vec{r}}=-e \nabla \phi(\vec{r}, t)
$$

where $M$ and $e$ are mass and charge of the ion. Putting potential (1) into equation of motion (2) yields:

$$
M \ddot{\vec{r}}+2 e f(t)(\vec{r}-3 \vec{z})=0
$$


where $\vec{z}=(0,0, z)$.

If we label:

$$
\psi=x, \quad \varphi=\frac{1}{2} \omega_{r f} t, \quad a=\frac{8 e U}{M \omega_{r f}^{2}\left(r_{0}^{2}+2 z_{0}^{2}\right)}, \quad q=\frac{4 e V}{M \omega_{r f}^{2}\left(r_{0}^{2}+2 z_{0}^{2}\right)},
$$

Eq.(3) may be rewritten in the form of the Mathieu equation [16]:

$$
\frac{\partial^{2} \psi(\varphi)}{d \varphi}+(a+2 q \cos (2 \varphi)) \psi(\varphi)=0
$$

The Mathieu equation is a well known equation of mathematical physics [17]], and it is usually encountered when studding the problems of parametric resonance [18, 19].

The presence of the areas of stable and unstable motion is a peculiarity of parametric resonance. The solutions of Eq. (5) may be as stable as unstable depending on the values of $a$ and $q$. In case of Paul trap, ion confinement inside a trap corresponds to stable solution. Because of this, we are interested in the stable solutions (2) of Eq. (5). Depending on physical situation for studying motion of ions inside Paul trap classical and quantum considerations are used. The modern state of affairs in studying this problem is given in [16]. In spite of the fact that, classical consideration of this problem is more or less adequate, quantum-mechanical consideration is far from perfection [16].

The goal of this work is to fill up this gap.

In this work we shall try to give more adequate (in our opinion) quantum mechanical description of motion of ions inside Paul trap. At the same time we shall use the results obtained by us from the study of the problem of quantum chaos [20, 21, 22, 23].

\section{STABLE QUANTUM-MECHANICAL SOLUTIONS. THE MATHIEU- SCHRODINGER EQUATION SYMMETRIES}

The Mathieu Eq.(5) in classical consideration, depending on the values of the parameters $a$ and $q$, has as stable as unstable solutions. On the plane $(a, q)$, the boundaries between stable and unstable solutions pass through special curve called the Mathieu characteristics [17]. Along these lines, to which certain set of the values of the parameters $(a(q), q)$ correspond, the well known periodic Mathieu functions $c e_{n}(\varphi, q), s_{n}(\varphi, q)$ [17] are solutions of classical Mathieu Eq.(5). Periodic solutions are the subject of our special interest, since periodic solutions corresponds to finite motion, which means confinement of ion inside Paul 
trap. It is worth noting that, this kind of selection of the values of the parameters of $a(q)$ and $q$ impose limitations on the amplitudes of constant and variable fields: $U$ and $V$ (see Eq.(4)).

In quantum consideration situation gets considerably complicated. The point is that, in quantum case Eq. (5) must be considered as the Mathieu-Schrodinger equation. In this case solution $\psi(\varphi, q)$ and the value of parameter $a(q)$ must be considered as eigenfunction and eigenvalue of quantum-mechanical equation. Characteristic property of the MathieuSchrodinger equation is the following: eigenfunctions $\psi_{n}(\varphi, q)$ can differ from the solutions of classical Mathieu equation $c e_{n}(\varphi, q), s e_{n}(\varphi, q)$. Finding of eigenfunctions $\psi_{n}(\varphi, q)$ of the Mathieu-Schrodinger equation is difficult problem and the application of group theory is needed. Interested reader can find details in work [21]. In the present work we shall only use the results needed further.

The eigenfunctions of the Mathieu-Schrodinger equation form the basis of irreducible representation of the Mathieu-Schrodinger equation symmetry group.

As is known [17], periodic solutions of Eq. (5) are given by the Mathieu functions:

$$
c e_{2 n}(\varphi, q), c e_{2 n+1}(\varphi, q), s e_{2 n+1}(\varphi, q), s e_{2 n+2}(\varphi, q)
$$

which setisfy the normalization condition:

$$
\frac{1}{\pi} \int_{0}^{2 \pi} \psi_{n}^{2}(\varphi, q) d \varphi=1
$$

where $\psi_{n}(\varphi, q)$ means Mathieu functions (6). To eigenfunctions (6) there corresponds the eigenvalues (Mathieu characteristics)

$$
a_{2 m}(q), \quad a_{2 m+1}(q), \quad b_{2 m+1}(q), \quad b_{2 m+2}(q),
$$

which also depend on the parameters $q$.

The properties of symmetry of the Mathieu functions can be presented in the form of Table (see Table 1 in Ref. [21]).

By immediate check it is easy to be convinced that symmetry properties of the Mathieu functions with respect to four elements of transformation:

$$
\begin{gathered}
G(\varphi \rightarrow-\varphi)=a, \quad G(\varphi \rightarrow \pi-\varphi)=b, \\
G(\varphi \rightarrow \pi+\varphi)=c, \quad G(\varphi \rightarrow \varphi)=e
\end{gathered}
$$


form a group. For this purpose it is enough to test the realization of the following relations:

$$
\begin{gathered}
a^{2}=b^{2}=c^{2}=e, \\
a b=c, \quad a c=b, \quad b c=a .
\end{gathered}
$$

Group $G$ contains three elements $a, b, c$ of the second order and unity element $e$. The group $G$ is isomorphic to the well-known group of Klein [24].

The group of transformations $G$ is not a simple group since it contains subgroups. When combined with the unit element, each of three elements $a, b, c$ forms a subgroup of second order:

$$
\begin{aligned}
& G_{+} \rightarrow e, b ; \\
& G_{-} \rightarrow e, c ; \\
& G_{0} \rightarrow e, a .
\end{aligned}
$$

Thus the Mathieu-Schrodinger equation is characterized by a presence of three symmetries determined by subgroups of symmetry group. Depending on the values of parameter $q$, the system can be in the states characterized by any of the symmetries (8). It is worth mention that, to the symmetries $G_{+}$and $G_{-}$correspond degenerate states with wave functions [21]:

$$
\begin{gathered}
G_{-} \rightarrow \psi_{2 n+1}^{ \pm}(\varphi)=\frac{\sqrt{2}}{2}\left(c e_{2 n+1}(\varphi) \pm i s e_{2 n+1}(\varphi)\right), \\
\psi_{2 n}^{ \pm}(\varphi)=\frac{\sqrt{2}}{2}\left(c e_{2 n}(\varphi) \pm i s e_{2 n}(\varphi)\right)
\end{gathered}
$$

and

$$
\begin{gathered}
G_{+} \rightarrow \eta_{2 n}^{ \pm}(\varphi)=\frac{\sqrt{2}}{2}\left(c e_{2 n}(\varphi) \pm i s e_{2 n+1}(\varphi)\right), \\
\eta_{2 n+1}^{ \pm}(\varphi)=\frac{\sqrt{2}}{2}\left(c e_{2 n+1}(\varphi) \pm i s e_{2 n+2}(\varphi)\right) .
\end{gathered}
$$

To the symmetry group $G_{0}$ correspond nondegenerate states with wave functions [21]:

$$
G_{0} \rightarrow c e_{2 n}(\varphi) ; c e_{2 n+1}(\varphi) ; s e_{2 n}(\varphi) ; s e_{2 n+1}(\varphi)
$$

In the end of this section, let us sum up some results.

The Mathieu-Schrodinger equation is characterized by a specific dependence of the spectrum of eigenvalues $a_{n}(q)$ and eigenfunctions $\psi_{n}(\varphi, q)$ on the parameter $q$ (see Fig.1). On the plane with the spectral characteristics (so-called Mathieu characteristics [17]) of the 
problem, this specific feature manifests itself in the alternation of areas of degenerate $\left(G_{ \pm}\right)$ and no degenerate $\left(G_{0}\right)$ states. The boundaries between these areas pass through the branch points of energy terms $a_{n}(q)$.

In case of quantum consideration, the problem of confinement of ion inside Paul trap, the aforesaid means the following:

In order ion's position to be localized inside Paul trap, it is necessary that the solutions of the Mathieu-Schrodinger quantum-mechanical equation to be periodic and as a result finite. This can be achieved only by proper selection of the values of the parameters $a(q)$, $q$ corresponding to the Mathieu characteristics, since along these lines motion is periodic. According to Eq. (4), this can be done by proper selection of the parameters of electric field $U$ and $V$. The system with these kind of selected values of the parameters can be found in the state characterized by one of the symmetry and it shall be described by corresponding wave functions (9)-(11) (see Fig.1).

In case of classical consideration the situation is different. Not only the Mathieu characteristics belong to the areas of stable motion but also the areas where the Floquet solutions are damped functions of time [25].

In case of quantum consideration the areas of damped solutions belong to forbidden energy zones in semiconductors [26]. This is characteristic property of quantum case.

\section{NONSTATIONARY CASE. STOCHASTIC HEATING OF THE SYSTEM}

Let us assume, that the amplitude of the electromagnetic field $V$ is modulated by a slowly changing field. According to (4), the influence of modulation can be taken into account by making a replacement in the Mathieu-Schrodinger equation (5)

$$
q(t)=q_{0}+\Delta q \cos \nu t
$$

where $\Delta q$ is the modulation amplitude expressed in dimensionless units and $\nu$ is the modulation frequency. In this case, the problem of ion's motion inside Paul trap is reduced to the problem studied in work [22]. In the mentioned work stochastic absorption of energy by non stationary chaotic quantum system was studied. Not going into details that can be found by interested reader in [22], here we only present the main results related to given problem.

After making replacement (12), Hamiltonian for the Mathieu-Schrodinger equation takes 
the form:

$$
\begin{gathered}
\hat{H}=\hat{H}_{0}+\hat{H}^{\prime}(t), \\
\hat{H}_{0}=-\frac{\partial^{2}}{\partial \varphi^{2}}+q_{0} \cos 2 \varphi, \\
\hat{H}^{\prime}(t)=\Delta q \cos 2 \varphi \cos \nu t .
\end{gathered}
$$

We assume that gradual change of $q(t)$ may involve some $\mathrm{N}$ branch points on the left and right side of the separatrix $a(q)=q$, (see Fig.1).

Simple calculations show that the matrix elements of perturbation $\hat{H}^{\prime}(t)$ with respect to the wave functions of the nondegenerate area $G_{0}(11)$ are equal to zero

$$
<c e_{n}\left|\hat{H}^{\prime}(t)\right| s e_{n}>=0 \text {. }
$$

But in the degenerate areas $G_{+}, G_{-}$

$$
H_{+-}^{\prime}=<\psi_{2 n+1}\left|\hat{H}^{\prime}(t)\right| \psi_{2 n+1}^{-}>\neq 0
$$

where $\psi_{2 n+1}^{ \pm}$are the functions $(9),(10)$ corresponding to the areas of symmetry $G_{-}$and $G_{+}$ (see Fig.1).

Thus the modulation (12) may cause transition between quantum states of the degenerate areas $G_{+}, G_{-}$.

When there is a small dispersion in the values of parameter $\delta q_{0}$, as was shown in work [22], mixed states are formed in the degenerate areas. As a result of this, the system may be with equal probability of $1 / 2$ in two states $\psi_{2 n}^{+}$and $\psi_{2 n}^{-}$. Consequently, because of specific dependence of energy terms $a_{n}(q)$ on the parameters $q$ (see Fig.1), and because of time modulation $q(t)$, the process is extended to other levels as well. At the same time absorption of energy by the system and stochastic heating takes place (see Fig. 8 of work [22]).

Something like that must take place in case of ion placed in Paul trap and kept there by amplitude modulated alternate electric field.

Thus Paul trap may be used for experimental observation of quantum chaos. The present theoretical analysis gives some recommendations concerning the values of dimensionless parameters of the Paul trap(4): $a \approx 6.7 \div 17.2 ; q_{0} \approx 12$.

The point is that, the problem of internal rotational motion in polyatomic molecules is reduced to the Mathieu-Schrodinger equation model [27]. Therefore as is shown in work 


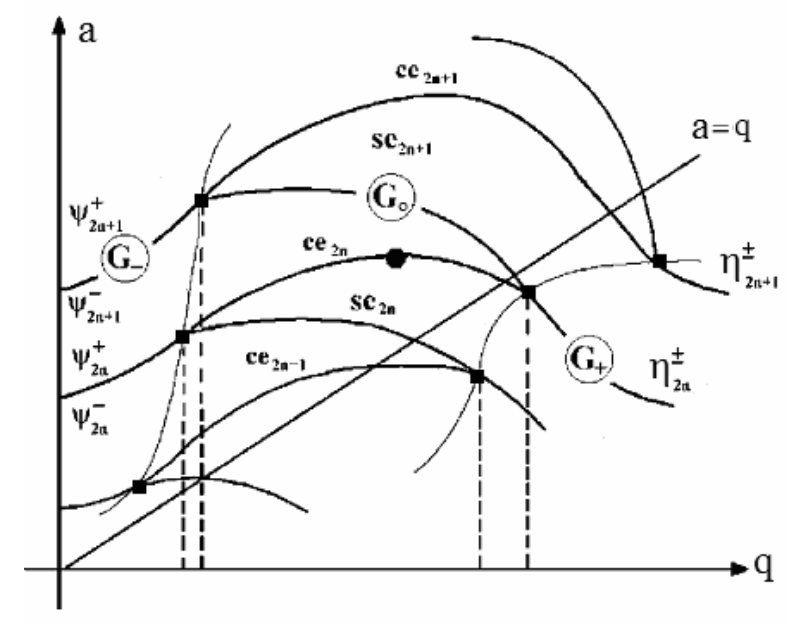

FIG. 1: A fragment of the parameter-dependent energy spectrum. Squares denote branch points.

[23], the present experimental data of infrared absorption in molecules $C_{2} H_{6}$ [28] are direct evidence of proposed model to be correct.

[1] M.H. Anderson, J.R. Ensher, M.R. Matthews, C.E. Wieman, and E.A.Cornell, Science 269, $198(1995)$.

[2] K.B. Davis, M.O. Mewes, M.R. Anrews, N.J.van Druten,D.S. Durfee, D.M. Kurn, and W. Ketterle, Phys. Rew. Lett. 75, 3969 (1995).

[3] C.C. Bradley, C.A. Sackett, J.J. Tollett, and R.G. Hulet, Phys. Rew. Lett. 75, 1687 (1995).

[4] D.S. Jin, J.R. Ensher, M.R. Matthews, C.E. Wieman, and E.A. Cornell, Phys. Rew. Lett. 77, 420 (1996).

[5] M.O. Mewes, M.R. Anrews, N.J. van Druten, D.M. Kurn,D.S. Durfee, and W. Ketterle, Phys. Rew. Lett. 77, 416 (1996).

[6] M.R. Anrews, M.O. Mewes, N.J. van Druten, D.S. Durfee, D.M. Kurn, and W. Ketterle, Science 273, 84 (1996).

[7] T. Aoki et al. Nature 443, 671-674 (2006).

[8] H. Mabuchi and A. Doherty, Science 298, 1372 (2002).

[9] C.J. Hood et al. Science 287, 1447 (2000).

[10] J. Raimond, M. Brune, and S. Haroche, Rev. Mod. Phys. 73, 565 (2001). 
[11] D.J. Wineland et al. J.Res. Nath. Inst. Stand.Technol.,103, 259 (1998).

[12] Q.A.Turchette et al. Phys. Rew. Lett. 75, 4710 (1995).

[13] W. Paul, Rev. Mod. Phys. 62, 531 (1990).

[14] R. Blatt, P.Gill, and R.C.Thomson, J. Mod.Opt. 39, 193 (1992).

[15] R. Blatt and W. Neuhauser, Appl.Phys. B. 59 (1994).

[16] W.P.Schleich, Quantum Optics in Phase Space, Wiley-VCH, Berlin (2001).

[17] M. Abramowitz and I. Stegun, Handbook of Mathematical Functions, US Government Printing Office, Washington, (1964).

[18] A.H. Nayfeh, Introduction to Perturbation Technques, Chichester, New York (1981).

[19] R.Z. Sagdeev, D.A. Usikov, and G.M. Zaslavsky, Nonlinear Physics,Harwood Academic, New York (1988).

[20] A. Ugulava, L.Chotorlishvili, and K.Nickoladze, Phys.Rev. E 68, 026216 (2003).

[21] A. Ugulava, L.Chotorlishvili, and K.Nickoladze, Phys.Rev. E 70, 026219 (2004).

[22] A. Ugulava, L.Chotorlishvili, and K.Nickoladze, Phys.Rev. E 71, 056211 (2005).

[23] A. Ugulava, L.Chotorlishvili, T. Gvarjaladze, and S.Chkhaidze, Mod.Phys. Lett. B, 21, 415 (2007).

[24] M. Hamermesh, Group Theory and its Application to Physical Problems, Addison-Wesley, Reading MA,(1964).

[25] H. Bateman and A. Erdelyi, Higher Transcendental Functions, McGraw-Hill, New York,v.3,(1995).

[26] R.E. Pierls, Quantum Theory of Solids, Oxford University Press, Oxford (1955).

[27] W.H. Flyger, Molecular Structure and Dynamics, New Jersy (1978).

[28] T. Shimanouchi, Tables of Molecular Vibrational Frequencies Consolidated, National Bureau of Standards 1, 1-160 (1972). 